\title{
Low-Concentration Measurements of Nuclear Spin-Induced Optical Rotation Using SABRE Hyperpolarization
}

\author{
Petr Štěpánek*(i) and Anu M. Kantola* \\ NMR Research Unit, Faculty of Science, University of Oulu, Oulu FI-90014, Finland \\ Supporting Information
}

ABSTRACT: Nuclear spin-induced optical rotation (NSOR) is a promising phenomenon for molecular structure elucidation due to its sensitivity to electronic structure near atomic nuclei. It is the only experimentally verified nuclear magneto-optic effect (NMOE), so far observed usually in neat liquids or in concentrated binary mixtures, with the proportion of the minor component at least $10 \%$. We report a method to extend the lower-concentration range of NSOR measurements by 2 orders of magnitude by employing continuous-flow SABRE (signal amplification by reversible exchange) hyperpolarization. This approach significantly increases the sensitivity of NSOR and enables its detection in dilute samples, as demonstrated with measurements of

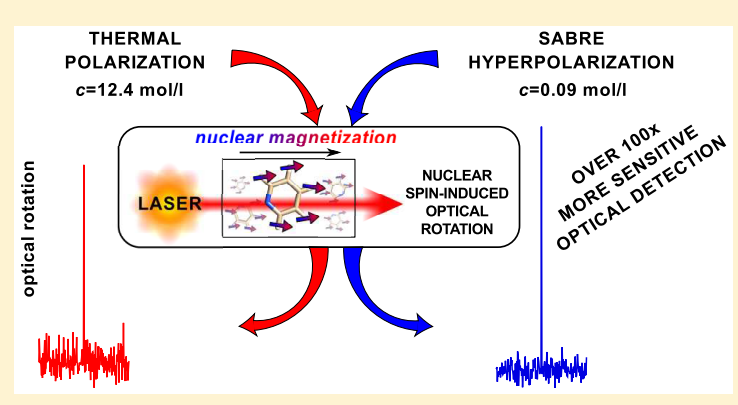
NSOR of $90 \mathrm{mmol} / \mathrm{L}$ solutions of pyridine and pyrazine. The results are compared with first-principles calculations, and good agreement is found. The possibility to measure low-concentration solutions significantly extends the pool of samples available for further studies of NMOEs.

$\mathrm{N}$ uclear magneto-optic effect (NMOE) is an umbrella term for a family of spectroscopic methods based on observing changes in the polarization state of a light beam after its interaction with molecules possessing a nonzero nuclear spin magnetization. Because the perturbations involve an electron cloud around the particular nucleus, the NMOEs promise to give new insights into the molecular structure with the resolution given by the local chemical environment, similarly as in nuclear magnetic resonance (NMR). Due to the relation to NMR, NMOEs contain information about detailed structural parameters, such as dipolar, quadrupolar, or recently experimentally verified spin-spin couplings ${ }^{1}$ or nuclear shielding. Moreover, the electromagnetic field of the light causes perturbations of the electron cloud that are not present in NMR and thus give rise to new observables. This is reflected in NMOEs as an additional aspect of the electronic structure and, for example, provides a selective response from groups involved in the electronic excitation. ${ }^{2,3}$

Out of the five described NMOEs, ${ }^{1-21}$ so far only nuclear spin-induced optical rotation (NSOR) has been observed for a range of molecules including water, ${ }^{4,6,12,13}$ organic liquids such as alcohols, ${ }^{12}$ hydrocarbons, ${ }^{6,12}$ halogenated hydrocarbons, ${ }^{6,7,12}$ organic phosphines, and thiols, ${ }^{7}$ and xenon. ${ }^{4}$ In general, these experiments share two common features: (a) in most cases, neat substances (pure liquids) were used as samples and (b) they were polarized thermally in a strong magnetic field. Notable exceptions are work of Pagliero et al., which probed binary mixtures of organic liquids with ratios as low as 1:9, and the work of Savukov et al., ${ }^{4}$ in which spinexchange optical pumping (SEOP) was used for hyperpolarization $(\mathrm{HP})$ of $\mathrm{Xe}$.
The choice of the sample of pure, i.e., highly concentrated, liquids is motivated by the rather low signal intensity of NSOR. This feature originates from low nuclear spin magnetization achievable via thermal polarization and limits the sensitivity of NSOR in the same way as in the traditional NMR. The signal intensity of NSOR (for a single type of chemically distinct nucleus $\mathrm{K}$ ) as a function of the incident light frequency $\nu$ can be expressed as

$$
\Theta(\nu)=\eta(\nu) c_{\mathrm{K}} l P_{\mathrm{K}}
$$

where $\Theta(\nu)$ is the rotation of the plane of polarization of the light in radians, $\eta(\nu)$ is a molecule-specific parameter (molar NSOR), $c_{\mathrm{K}}$ is the concentration of the observed nucleus $\mathrm{K}, l$ is the length of the sample traversed by the light beam, and $P_{\mathrm{K}} \in$ $[0 ; 1]$ is the fractional magnetization of the nucleus $\mathrm{K}$ along the light beam. As $\eta(\nu)$ is a molecule-specific constant, the signal can be increased by varying only the remaining three parameters: concentration, length of the optical path, and spin polarization. Increasing the concentration $c_{\mathrm{K}}$ is the most straightforward approach and justifies the use of pure substances in the previous studies. However, it is of limited use, for example, when investigating concentration dependence, chemical equilibria, or when the sample is prohibitively expensive or simply is not in a liquid form by itself and has low solubility. The optical path $l$ can be varied rather freely, assuming that the optical properties of the sample allow it. As an example, the increase of path has been exploited in previous

Received: July 26, 2019

Accepted: August 27, 2019

Published: August 27, 2019 
works using multiple passes through a long cell, bringing the total length to over $3 \mathrm{~m} .{ }^{12}$

A very promising way how to increase the NSOR signal is through the polarization $P$, which in thermally polarized samples, such as those produced in standard high-field NMR magnets, is on the order of about $\sim 10^{-5}$. Because small values of $P$ are a nuisance in standard NMR and MRI as well, the socalled HP techniques have been heavily investigated in recent years. Thanks to these advances, it is now possible to boost the signal by several orders of magnitude to achieve polarization levels of up to tens of percent. ${ }^{22-28}$ The use of HP was demonstrated in NSOR for the case of Xe polarized via SEOP. ${ }^{4}$ However, SEOP is limited solely to HP of noble gases.

In this work, we demonstrate an increase in the NSOR signal by employing the signal amplification by reversible exchange (SABRE) technique to boost the nuclear polarization. SABRE is a solution-based technique based on a reversible exchange of para-hydrogen gas and the target molecule at a metal catalyst center, which facilitate transfer of spin order from parahydrogen to the substrate being hyperpolarized. SABRE has been shown to be a powerful method for several nuclei, including ${ }^{1} \mathrm{H},{ }^{13} \mathrm{C},{ }^{15} \mathrm{~N},{ }^{19} \mathrm{~F},{ }^{31} \mathrm{P},{ }^{29} \mathrm{Si}$, and ${ }^{119} \mathrm{Sn} .{ }^{29-50}$ Its further advantages compared to the other $\mathrm{HP}$ techniques is its low cost and relative versatility accommodating for a range of different substrates.

The NSOR was measured in a low-field custom-built system, schematically depicted in Figure 1. The sample, which was

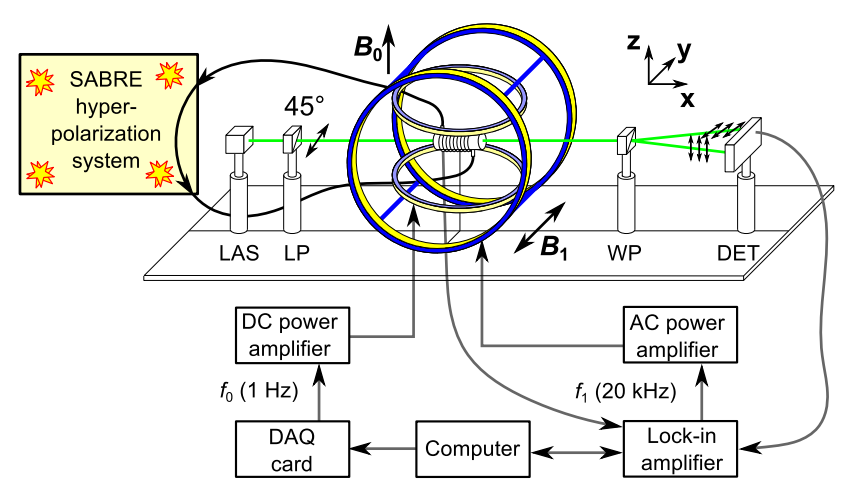

Figure 1. Sketch of the NSOR system. The laser beam produced by a diode laser (LAS, 520 or $690 \mathrm{~nm}$, Thorlabs) is polarized to $45^{\circ}$ via linear polarizer (LP) and passes through the sample in a $7 \mathrm{~cm}$ long flow-through cell. The resulting beam is analyzed by Wollaston prism (WP) and balanced detector. The spin polarization is produced in a SABRE polarizer, ${ }^{51}$ which continuously supplies fresh sample to the cell. The magnetization precesses along the $B_{0}(z)$ field, which is modulated with a frequency of $1 \mathrm{~Hz}$ to bring it in and out of resonance with continuous spin-locking field $B_{1}(y)$. The magnetization is thus periodically spin-locked in precession around the $z$-axis in the $x y$-plane or resting along the $B_{0}$. See the SI for details.

produced in a SABRE polarizer ${ }^{51}$ interfaced with the optical system, was continuously pumped into a cylindrical glass cell (length $70 \mathrm{~mm}$, inner/outer diameter $10 \mathrm{~mm} / 13 \mathrm{~mm}$ ) inside of a custom-made electromagnet coil system (Serviciencia, S.L.). Thermally polarized sample prepared by placing a container in a superconducting $14.1 \mathrm{~T}(600 \mathrm{MHz})$ NMR magnet was measured for reference. Because the sample in SABRE experiments contains salts and organic solvents, it is preferable to isolate the pumping mechanism from contact with the sample itself. This motivated us to use a peristaltic pump, which, however, has usually rather limited available flow rates. For these reasons, we opted for a smaller cell with a shorter path length $(7 \mathrm{~cm})$ compared to previous experiments and compensated for this by longer measurement time.

The spin-polarized sample was subjected to two magnetic fields: an alternating field $B_{1}$ in the $y$-direction (perpendicular to the axis of the cell) and a static $B_{0}$ field in the $z$-direction (vertical), which is a sum of a stray field from the $600 \mathrm{MHz}$ NMR magnet $(0.12 \mathrm{mT})$ and a field generated by the electromagnet $(0.37 \mathrm{mT})$. The $B_{0}$ field was modulated at a frequency of $1 \mathrm{~Hz}$ using a slow ramp, to bring the proton Larmor frequency in and out of resonance with the $B_{1}$ field. The signal was thus modulated at a frequency of $1 \mathrm{~Hz}$ (see the Methods Section and SI for more details).

The obtained values were compared with the NSOR calculated using the BHandHLYP density functional and coupled cluster singles and doubles (CCSD) with three completeness optimized basis sets ${ }^{52} \operatorname{co} 0,{ }^{9} \operatorname{co} 2,{ }^{12,53}$ and $\operatorname{co} 2$ MOR. ${ }^{18}$ Theoretical results were corrected for the local effects of the medium on the optical field and for the Faraday rotation arising from the long-range effects of the magnetized bulk.

The results of the measurements are shown in Figure 2. Three experiments were conducted: (a) NSOR of thermally polarized neat pyridine; (b) NSOR of a pyridine solution in methanol, polarized using SABRE; and (c) NSOR of a solution of pyrazine in methanol, polarized using SABRE. The NSOR signals normalized for concentration, length of the optical path, and nuclear polarization and calculated values corrected for the medium effects ${ }^{8,9}$ (see the SI for details) are reported in Table 1 . The data were obtained by averaging a total of $16000 \mathrm{~s}$ of measurement.

Figure 2a shows the NSOR signal of thermally polarized neat pyridine $\left(c_{\text {pyridine }}=12.4 \mathrm{~mol} / \mathrm{L}\right)$ as a function of the $B_{0}$ modulation. The sharp peak at $1 \mathrm{~Hz}$ clearly indicates the presence of an observable NSOR signal. To affirm that the signal is not caused by any interference, a baseline measurement was performed where the container with the pyridine was lowered below the NMR magnet, placing it in a comparatively negligible magnetic field and thus removing the thermal polarization. Under these circumstances, the peak at $1 \mathrm{~Hz}$ could no longer be observed, indicating its origin in nuclear magnetization (see the SI).

Figure $2 \mathrm{~b}$ shows the NSOR signal of pyridine $\left(c_{\text {pyridine }}=90\right.$ $\mathrm{mmol} / \mathrm{L}$ ), in methanol polarized using SABRE. The NSOR signal stemming from the hyperpolarized pyridine can be clearly observed. The intensity is slightly larger compared to the case of thermally polarized pyridine, in line with the increase of nuclear polarization as observed with NMR. It is worth noting that the NSOR intensities are not directly proportional to the increase in NMR signal due to contribution of the Faraday effect, which is considerably larger in pyridine than that in methanol.

The results for the third sample, the solution of pyrazine $\left(c_{\text {pyrazine }}=86 \mathrm{mmol} / \mathrm{L}\right)$, are shown in Figure $2 \mathrm{c}$. Note that a laser of a different wavelength $(\lambda=690 \mathrm{~nm})$ compared to the above experiments was used due to the optical properties of the solution. The NSOR signal is again clearly visible above the noise level, with the statistical significance safely exceeding 3 standard deviations (see Table 1). This result demonstrates the main benefit of our approach, i.e., the possibility to observe NSOR from molecules that are at standard conditions solid and thus require being measured in solution.

It might be noticed that the level of noise between different experiments varies. This can be explained by use of different 


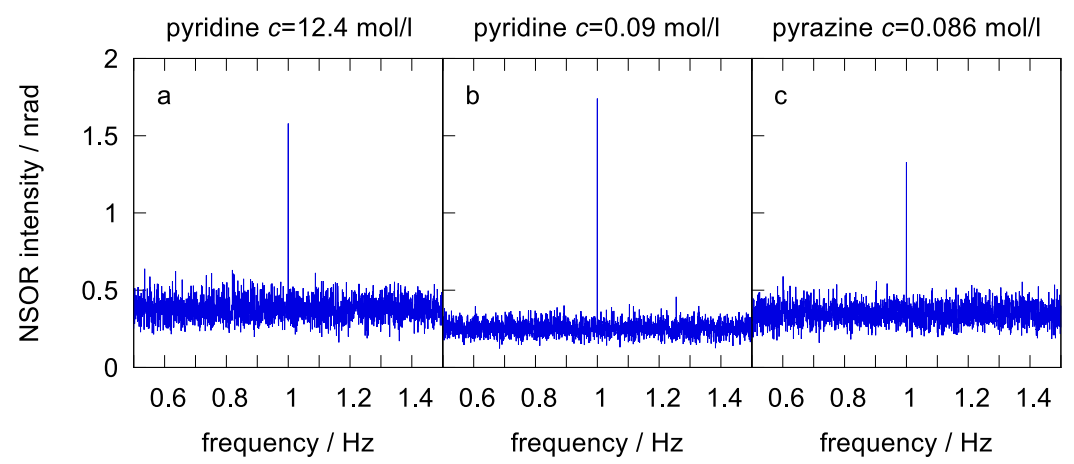

Figure 2. Plots of measured NSOR signals for pyridine and pyrazine using either thermal or SABRE polarization. Left pane: thermally polarized neat pyridine $\left(c_{\text {pyridine }}=12.4 \mathrm{~mol} / \mathrm{L}\right)$; middle pane: SABRE-polarized solution of pyridine $\left(c_{\text {pyridine }}=0.09 \mathrm{~mol} / \mathrm{L}\right)$ in methanol; right pane: SABREpolarized solution of pyrazine $\left(c_{\text {pyrazine }}=0.086 \mathrm{~mol} / \mathrm{L}\right)$ in methanol.

Table 1. Summary of the Results and Relevant Properties of the Three Measured Samples, Showing Concentration $c$, Measured NMR Signals in nA, Calculated Average Proton Polarization $P_{\mathrm{H}}$, Measured Optical Rotation Reported with $\pm 3 \sigma$, Normalized Experimental NSOR, and Theoretically Calculated NSOR Using CCSD/co2-MOR, Including Corrections for Bulk Properties $^{a}$

$\begin{array}{ccccccc}\text { sample } & c(\mathrm{~mol} / \mathrm{L}) & \mathrm{NMR} \text { current }(\mathrm{nA}) & P_{\mathrm{H}}(\%) & \Theta(\mathrm{nrad}) & \mathrm{NSOR}(\exp )\left(\mu \mathrm{rad} \cdot \mathrm{mol}^{-1} \cdot \mathrm{L} \cdot \mathrm{cm}^{-1}\right) & \mathrm{NSOR}(\mathrm{calc})\left(\mu \mathrm{rad} \cdot \mathrm{mol}{ }^{-1} \cdot \mathrm{L} \cdot \mathrm{cm}^{-1}\right) \\ \text { pyridine (thermal) } & 12.4 & 475 & 1.56 \times 10^{-3} & 1.53 \pm 0.21 & 0.87 & 1.03 \\ \text { pyridine (SABRE) } & 0.090 & 718 & 0.324 & 1.72 \pm 0.14 & 0.65 & 0.80 \\ \text { pyrazine (SABRE) } & 0.086 & 881 & 0.521 & 1.28 \pm 0.19 & 0.39 & 0.47\end{array}$

${ }^{a}$ NSOR values are reported as the average per proton. Further results from calculations using different methods are shown in the SI.

laser sources and as an effect of different properties of the solution, such as viscosity and the amount of dissolved gases, which affect the flow and the bubble formation in the liquid during the pumping.

The results for NMR signals and experimental as well as calculated NSOR intensities are summarized in Table 1 together with key sample properties. NMR intensities of comparable magnitude can be observed in all three samples, despite 2 orders of magnitude difference in their concentrations, confirming the presence of a hyperpolarized sample. Accordingly, the NSOR signals are of similar intensity.

It can also be seen that the experimentally obtained NSOR signals compare rather well with the ones from quantum chemical calculations. The differences can be attributed to the common features of the first-principle calculations of properties of moderately sized molecules in the condensed phase, such as an approximate wave function (or density), basis set effects, and the absence of rovibrational corrections and explicit solvent-solute interactions. The calculated values were corrected for the average effect of the solvent on the optical field and for the Faraday rotation caused by magnetization of the sample. It is noteworthy that the latter correction accounts for the difference between NSOR of pyridine in neat liquid and that in methanol as pyridine has about a 3 times larger Verdet constant and thus more significant contribution to the rotation. This effect is reproduced in the calculated results.

The total NSOR signals are on a similar scale, albeit somewhat weaker, compared to protons in other organic molecules reported earlier. ${ }^{12}$ The overall weaker signal is in large part due to the longer laser wavelength compared to the previously used $405 \mathrm{~nm}$ as the NSOR signal is decreasing with wavelength. ${ }^{4,9}$ The shift in wavelength is also the cause of most of the observed difference in NSOR between pyridine and pyrazine as quantum chemical calculations show that at the same wavelength the signals would be of similar magnitude.
We have presented a new approach to measure NSOR at low concentrations by employing continuous polarization with the SABRE method and demonstrated the utility of the technique on measurements of dilute solutions of pyridine and pyrazine. The approach allows one to measure samples at concentrations by at least 2 orders of magnitude lower compared to neat substances employed in the majority of previous studies. Notably, we demonstrated the feasibility of NSOR measurements of solid substances by a combination of dissolution and HP. Although SABRE is not as general of a method for HP as DNP (dynamic nuclear polarization), the setup can be constructed with only modest financial investments and the preparation of the sample is repeatable, rather fast, and can be implemented in continuous mode. The presented methodology opens a way to significantly broaden the range of molecules accessible for NSOR measurements and development of deeper understanding in this new emerging field of spectroscopy.

\section{METHODS SECTION}

The laser beam is supplied by a 520 or $690 \mathrm{~nm}$ laser diode (Thorlabs) and is passed through a linear polarizer, which prepares the light polarized at $45^{\circ}$ with respect to the vertical plane. The light beam passes through the sample, acquires optical rotation, and is analyzed by a Wollaston prism and difference detector. Due to the flow of the liquid and formation of small bubbles, the light intensity is diminished from the initial value by about $30 \%$ ( $50 \%$ in the case of pyridine/SABRE due to slight absorption by the SABRE catalyst). The signal from the optical detector is fed into a lock-in amplifier (MFLI, Zurich Instruments) tuned to the frequency of $B_{1}$. Because the envelope of the measured signal is modulated at the frequency of the $B_{0}$ field, the signal from the optical detector is Fourier transformed and the NSOR signal appears at the $B_{0}$ modulation frequency (i.e., $1 \mathrm{~Hz}$ ). To obtain the degree of nuclear magnetization, a reference NMR signal was measured 
as a current induced in a solenoid coil wound around the sample cell (140 turns, 4 layers, $R=8.6 \Omega, L=1.139 \mathrm{mH}$ ). The NMR circuit was kept open during the measurement of NSOR to avoid induction of an extraneous magnetic field, which would give rise to Faraday rotation artifacts. The instrument control and data acquisition were done using custom LabVIEW programs.

\section{ASSOCIATED CONTENT}

\section{S Supporting Information}

The Supporting Information is available free of charge on the ACS Publications website at DOI: 10.1021/acs.jpclett.9b02194.

Experimental details of sample preparation and results of theoretical calculations (PDF)

\section{AUTHOR INFORMATION}

\section{Corresponding Authors}

*E-mail: petr.stepanek@oulu.fi.

*E-mail: anu.kantola@oulu.fi.

ORCID $\odot$

Petr Šěpánek: 0000-0001-5000-8180

Notes

The authors declare no competing financial interest.

\section{ACKNOWLEDGMENTS}

This project received funding from the European Union's Horizon 2020 research and innovation programme under the Marie Skłodowska-Curie grant agreement NMOSPEC, No.

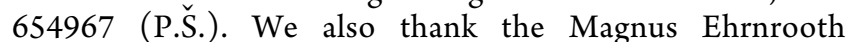
Foundation for financial support (P.S.). The authors acknowledge financial support from the Kvantum institute (University of Oulu) and from the Academy of Finland (Grant 316180) (P.S.). We acknowledge grants of computer capacity from the Finnish Grid and Cloud Infrastructure (persistent identifier urn:nbn:fi:research-infras-2016072533). The authors thank Anne Selent and Marcin Selent for valuable discussions of the optical system setup and manufacturing of several components, Jouni Sarala for construction of the circuit board for the NMR system, Vladimir Zhivonitko for synthesis of the SABRE catalyst, and Juha Vaara for discussions about bulk corrections of computational results.

\section{REFERENCES}

(1) Zhu, Y.; Gao, Y.; Rodocker, S.; Savukov, I.; Hilty, C. Multinuclear Detection of Nuclear Spin Optical Rotation at Low Field. J. Phys. Chem. Lett. 2018, 9, 3323-3327.

(2) Ikäläinen, S.; Romalis, M. V.; Lantto, P.; Vaara, J. Chemical Distinction by Nuclear Spin Optical Rotation. Phys. Rev. Lett. 2010, 105, 153001.

(3) Štěpánek, P.; Coriani, S. Spatial localization in nuclear spininduced circular dichroism - a quadratic response function analysis. Phys. Chem. Chem. Phys. 2019, 21, 18082-18091.

(4) Savukov, I. M.; Lee, S.; Romalis, M. V. Optical detection of liquid-state NMR. Nature 2006, 442, 1021-1024.

(5) Lu, T.; He, M.; Chen, D.; He, T.; Liu, F. Nuclear-spin-induced optical Cotton-Mouton effect in fluids. Chem. Phys. Lett. 2009, 479, 14-19.

(6) Pagliero, D.; Dong, W.; Sakellariou, D.; Meriles, C. A. Timeresolved, optically detected NMR of fluids at high magnetic field. J. Chem. Phys. 2010, 133, 154505.
(7) Pagliero, D.; Meriles, C. A. Magneto-optical contrast in liquidstate optically detected NMR spectroscopy. Proc. Natl. Acad. Sci. U. S. A. 2011, 108, 19510-19515.

(8) Yao, G.; He, M.; Chen, D.; He, T.; Liu, F. Analytical theory of the nuclear-spin-induced optical rotation in liquids. Chem. Phys. 2011, 387, 39-47.

(9) Pennanen, T. S.; Ikäläinen, S.; Lantto, P.; Vaara, J. Nuclear spin optical rotation and Faraday effect in gaseous and liquid water. $J$. Chem. Phys. 2012, 136, 184502.

(10) Ikäläinen, S.; Lantto, P.; Vaara, J. Fully Relativistic Calculations of Faraday and Nuclear Spin-Induced Optical Rotation in Xenon. J. Chem. Theory Comput. 2012, 8, 91-98.

(11) Yao, G.; He, M.; Chen, D.; He, T.; Liu, F. New Nuclear-SpinInduced Cotton-Mouton Effect in Fluids at High DC Magnetic Field. ChemPhysChem 2012, 13, 1325-1331.

(12) Shi, J.; Ikäläinen, S.; Vaara, J.; Romalis, M. V. Observation of Optical Chemical Shift by Precision Nuclear Spin Optical Rotation Measurements and Calculations. J. Phys. Chem. Lett. 2013, 4, 437441.

(13) Savukov, I. M.; Chen, H.; Karaulanov, T.; Hilty, C. Method for accurate measurements of nuclear-spin optical rotation for applications in correlated optical-NMR spectroscopy. J. Magn. Reson. 2013, 232, 31-38.

(14) Fu, L.; Vaara, J. Nuclear spin-induced Cotton-Mouton effect in molecules. J. Chem. Phys. 2013, 138, 204110.

(15) Fu, L.; Rizzo, A.; Vaara, J. Communication: Nuclear quadrupole moment-induced Cotton-Mouton effect in noble gas atoms. J. Chem. Phys. 2013, 139, 181102.

(16) Fu, L.; Vaara, J. Nuclear quadrupole moment-induced CottonMouton effect in molecules. J. Chem. Phys. 2014, 140, 024103.

(17) Fu, L.; Vaara, J. Nuclear-Spin-Induced Cotton-Mouton Effect in a Strong External Magnetic Field. ChemPhysChem 2014, 15, 23372350.

(18) Vähäkangas, J.; Lantto, P.; Vaara, J. Faraday Rotation in Graphene Quantum Dots: Interplay of Size, Perimeter Type, and Functionalization. J. Phys. Chem. C 2014, 118, 23996-24005.

(19) Vaara, J.; Rizzo, A.; Kauczor, J.; Norman, P.; Coriani, S. Nuclear spin circular dichroism. J. Chem. Phys. 2014, 140, 134103.

(20) Straka, M.; Štěpánek, P.; Coriani, S.; Vaara, J. Nuclear spin circular dichroism in fullerenes: a computational study. Chem. Commun. 2014, 50, 15228-15231.

(21) Štěpánek, P.; Coriani, S.; Sundholm, D.; Ovchinnikov, V. A.; Vaara, J. Relation between molecular electronic structure and nuclear spin-induced circular dichroism. Sci. Rep. 2017, 7, 46617.

(22) Ardenkjær-Larsen, J. H.; Fridlund, B.; Gram, A.; Hansson, G.; Hansson, L.; Lerche, M. H.; Servin, R.; Thaning, M.; Golman, K. Increase in signal-to-noise ratio of $>10,000$ times in liquid-state NMR. Proc. Natl. Acad. Sci. U. S. A. 2003, 100, 10158-10163.

(23) Barskiy, D. A.; Coffey, A. M.; Nikolaou, P.; Mikhaylov, D. M.; Goodson, B. M.; Branca, R. T.; Lu, G. J.; Shapiro, M. G.; Telkki, V.V.; Zhivonitko, V. V.; et al. NMR Hyperpolarization Techniques of Gases. Chem. - Eur. J. 2017, 23, 725-751.

(24) Nikolaou, P.; Coffey, A. M.; Walkup, L. L.; Gust, B. M.; Whiting, N.; Newton, H.; Barcus, S.; Muradyan, I.; Dabaghyan, M.; Moroz, G. D.; et al. Near-unity nuclear polarization with an opensource Xe-129 hyperpolarizer for NMR and MRI. Proc. Natl. Acad. Sci. U. S. A. 2013, 110, 14150-14155.

(25) Bowers, C. R.; Weitekamp, D. P. Parahydrogen and synthesis allow dramatically enhanced nuclear alignment. J. Am. Chem. Soc. 1987, 109, 5541-5542.

(26) Eisenschmid, T. C.; Kirss, R. U.; Deutsch, P. P.; Hommeltoft, S. I.; Eisenberg, R.; Bargon, J.; Lawler, R. G.; Balch, A. L. Para hydrogen induced polarization in hydrogenation reactions. J. Am. Chem. Soc. 1987, 109, 8089-8091.

(27) Pravica, M. G.; Weitekamp, D. P. Net NMR alignment by adiabatic transport of parahydrogen addition products to high magnetic field. Chem. Phys. Lett. 1988, 145, 255-258.

(28) Rayner, P. J.; Burns, M. J.; Olaru, A. M.; Norcott, P.; Fekete, M.; Green, G. G. R.; Highton, L. A. R.; Mewis, R. E.; Duckett, S. B. 
Delivering strong ${ }^{1} \mathrm{H}$ nuclear hyperpolarization levels and long magnetic lifetimes through signal amplification by reversible exchange. Proc. Natl. Acad. Sci. U. S. A. 2017, 114, E3188-E3194.

(29) Adams, R. W.; Aguilar, J. A.; Atkinson, K. D.; Cowley, M. J.; Elliott, P. I. P.; Duckett, S. B.; Green, G. G. R.; Khazal, I. G.; LópezSerrano, J.; Williamson, D. C. Reversible Interactions with paraHydrogen Enhance NMR Sensitivity by Polarization Transfer. Science 2009, 323, 1708-1711.

(30) Atkinson, K. D.; Cowley, M. J.; Elliott, P. I. P.; Duckett, S. B.; Green, G. G. R.; López-Serrano, J.; Whitwood, A. C. Spontaneous Transfer of Parahydrogen Derived Spin Order to Pyridine at Low Magnetic Field. J. Am. Chem. Soc. 2009, 131, 13362-13368.

(31) Atkinson, K. D.; Cowley, M. J.; Duckett, S. B.; Elliott, P. I. P.; Green, G. G. R.; López-Serrano, J.; Khazal, I. G.; Whitwood, A. C. Para-Hydrogen Induced Polarization without Incorporation of ParaHydrogen into the Analyte. Inorg. Chem. 2009, 48, 663-670.

(32) Zeng, H.; Xu, J.; Gillen, J.; McMahon, M. T.; Artemov, D.; Tyburn, J.-M.; Lohman, J. A.; Mewis, R. E.; Atkinson, K. D.; Green, G. G. R.; et al. Optimization of SABRE for polarization of the tuberculosis drugs pyrazinamide and isoniazid. J. Magn. Reson. 2013, 237, 73-78.

(33) Eshuis, N.; Hermkens, N.; van Weerdenburg, B. J. A.; Feiters, M. C.; Rutjes, F. P. J. T.; Wijmenga, S. S.; Tessari, M. Toward Nanomolar Detection by NMR Through SABRE Hyperpolarization. J. Am. Chem. Soc. 2014, 136, 2695-2698.

(34) Hövener, J.-B.; Schwaderlapp, N.; Borowiak, R.; Lickert, T.; Duckett, S. B.; Mewis, R. E.; Adams, R. W.; Burns, M. J.; Highton, L. A. R.; Green, G. G. R; et al. Toward Biocompatible Nuclear Hyperpolarization Using Signal Amplification by Reversible Exchange: Quantitative in Situ Spectroscopy and High-Field Imaging. Anal. Chem. 2014, 86, 1767-1774.

(35) Coffey, A. M.; Kovtunov, K. V.; Barskiy, D. A.; Koptyug, I. V.; Shchepin, R. V.; Waddell, K. W.; He, P.; Groome, K. A.; Best, Q. A.; Shi, F.; et al. High-Resolution Low-Field Molecular Magnetic Resonance Imaging of Hyperpolarized Liquids. Anal. Chem. 2014, 86, 9042-9049.

(36) Mewis, R. E.; Atkinson, K. D.; Cowley, M. J.; Duckett, S. B.; Green, G. G. R.; Green, R. A.; Highton, L. A. R.; Kilgour, D.; Lloyd, L. S.; Lohman, J. A. B.; et al. Probing signal amplification by reversible exchange using an NMR flow system. Magn. Reson. Chem. 2014, 52, 358-369.

(37) Mewis, R. E.; Green, R. A.; Cockett, M. C. R.; Cowley, M. J.; Duckett, S. B.; Green, G. G. R.; John, R. O.; Rayner, P. J.; Williamson, D. C. Strategies for the Hyperpolarization of Acetonitrile and Related Ligands by SABRE. J. Phys. Chem. B 2015, 119, 1416-1424.

(38) Pravdivtsev, A. N.; Yurkovskaya, A. V.; Zimmermann, H.; Vieth, H.-M.; Ivanov, K. L. Transfer of SABRE-derived hyperpolarization to spin- $1 / 2$ heteronuclei. RSC $A d v$. 2015, 5, 6361563623.

(39) Theis, T.; Truong, M. L.; Coffey, A. M.; Shchepin, R. V.; Waddell, K. W.; Shi, F.; Goodson, B. M.; Warren, W. S.; Chekmenev, E. Y. Microtesla SABRE Enables 10\% Nitrogen-15 Nuclear Spin Polarization. J. Am. Chem. Soc. 2015, 137, 1404-1407.

(40) Truong, M. L.; Theis, T.; Coffey, A. M.; Shchepin, R. V.; Waddell, K. W.; Shi, F.; Goodson, B. M.; Warren, W. S.; Chekmenev, E. Y. ${ }^{15} \mathrm{~N}$ Hyperpolarization by Reversible Exchange Using SABRESHEATH. J. Phys. Chem. C 2015, 119, 8786-8797.

(41) Barskiy, D. A.; Shchepin, R. V.; Coffey, A. M.; Theis, T.; Warren, W. S.; Goodson, B. M.; Chekmenev, E. Y. Over $20 \%{ }^{15} \mathrm{~N}$ Hyperpolarization in Under One Minute for Metronidazole, an Antibiotic and Hypoxia Probe. J. Am. Chem. Soc. 2016, 138, 80808083.

(42) Logan, A. W. J.; Theis, T.; Colell, J. F. P.; Warren, W. S.; Malcolmson, S. J. Hyperpolarization of Nitrogen-15 Schiff Bases by Reversible Exchange Catalysis with para-Hydrogen. Chem. - Eur. J. 2016, 22, 10777-10781.

(43) Kovtunov, K. V.; Kovtunova, L. M.; Gemeinhardt, M. E.; Bukhtiyarov, A. V.; Gesiorski, J.; Bukhtiyarov, V. I.; Chekmenev, E. Y.; Koptyug, I. V.; Goodson, B. M. Heterogeneous Microtesla SABRE
Enhancement of ${ }^{15} \mathrm{~N}$ NMR Signals. Angew. Chem., Int. Ed. 2017, 56, 10433-10437.

(44) Colell, J. F. P.; Logan, A. W. J.; Zhou, Z.; Shchepin, R. V.; Barskiy, D. A.; Gerardo, X.; Ortiz, J.; Wang, Q.; Malcolmson, S. J.; Chekmenev, E. Y.; Warren, W. S.; et al. Generalizing, Extending, and Maximizing Nitrogen-15 Hyperpolarization Induced by Parahydrogen in Reversible Exchange. J. Phys. Chem. C 2017, 121, 6626-6634.

(45) Colell, J. F. P.; Emondts, M.; Logan, A. W. J.; Shen, K.; Bae, J.; Shchepin, R. V.; Gerardo, X.; Ortiz, J.; Spannring, P.; Wang, Q.; Malcolmson, S. J.; et al. Direct Hyperpolarization of Nitrogen-15 in Aqueous Media with Parahydrogen in Reversible Exchange. J. Am. Chem. Soc. 2017, 139, 7761-7767.

(46) Shchepin, R. V.; Goodson, B. M.; Theis, T.; Warren, W. S.; Chekmenev, E. Y. Toward Hyperpolarized ${ }^{19} \mathrm{~F}$ Molecular Imaging via Reversible Exchange with Parahydrogen. ChemPhysChem 2017, 18, 1961-1965.

(47) Olaru, A. M.; Robertson, T. B. R.; Lewis, J. S.; Antony, A.; Iali, W.; Mewis, R. E.; Duckett, S. B. Extending the Scope of ${ }^{19} \mathrm{~F}$ Hyperpolarization through Signal Amplification by Reversible Exchange in MRI and NMR Spectroscopy. ChemistryOpen 2018, 7, 97-105.

(48) Burns, M. J.; Rayner, P. J.; Green, G. G. R.; Highton, L. A. R.; Mewis, R. E.; Duckett, S. B. Improving the Hyperpolarization of ${ }^{31} \mathrm{P}$ Nuclei by Synthetic Design. J. Phys. Chem. B 2015, 119, 5020-5027.

(49) Zhivonitko, V. V.; Skovpin, I. V.; Koptyug, I. V. Strong ${ }^{31} \mathrm{P}$ nuclear spin hyperpolarization produced via reversible chemical interaction with parahydrogen. Chem. Commun. 2015, 51, 25062509.

(50) Olaru, A. M.; Burt, A.; Rayner, P. J.; Hart, S. J.; Whitwood, A. C.; Green, G. G. R.; Duckett, S. B. Using signal amplification by reversible exchange (SABRE) to hyperpolarise ${ }^{119} \mathrm{Sn}$ and ${ }^{29} \mathrm{Si}$ NMR nuclei. Chem. Commun. 2016, 52, 14482-14485.

(51) Stěpánek, P.; Sanchez-Perez, C.; Telkki, V.-V.; Zhivonitko, V. V.; Kantola, A. M. High-throughput continuous-flow system for SABRE hyperpolarization. J. Magn. Reson. 2019, 300, 8-17.

(52) Manninen, P.; Vaara, J. Systematic Gaussian basis-set limit using completeness-optimized primitive sets. A case for magnetic properties. J. Comput. Chem. 2006, 27, 434-445.

(53) Ikäläinen, S.; Lantto, P.; Manninen, P.; Vaara, J. Laser-induced nuclear magnetic resonance splitting in hydrocarbons. J. Chem. Phys. 2008, 129, 124102. 